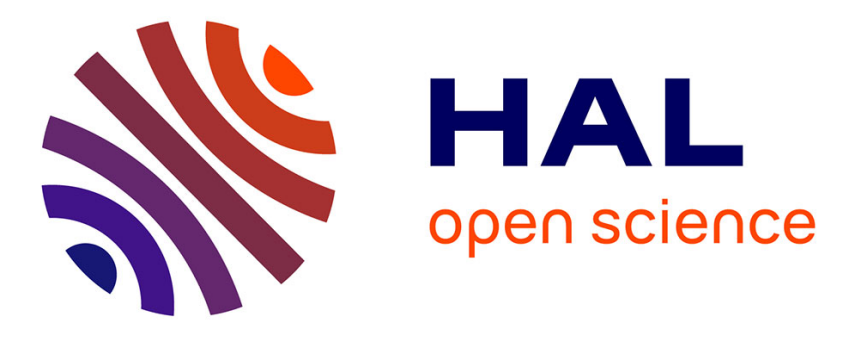

\title{
Two-Level Checkpointing and Verifications for Linear Task Graphs
}

\author{
Anne Benoit, Aurélien Cavelan, Yves Robert, Hongyang Sun
}

\section{To cite this version:}

Anne Benoit, Aurélien Cavelan, Yves Robert, Hongyang Sun. Two-Level Checkpointing and Verifications for Linear Task Graphs. The 17th IEEE International Workshop on Parallel and Distributed Scientific and Engineering Computing (PDSEC 2016), May 2016, Chicago, United States. pp.10, 10.1109/IPDPSW.2016.106 . hal-01354625

\section{HAL Id: hal-01354625 \\ https://hal.inria.fr/hal-01354625}

Submitted on 19 Aug 2016

HAL is a multi-disciplinary open access archive for the deposit and dissemination of scientific research documents, whether they are published or not. The documents may come from teaching and research institutions in France or abroad, or from public or private research centers.
L'archive ouverte pluridisciplinaire HAL, est destinée au dépôt et à la diffusion de documents scientifiques de niveau recherche, publiés ou non, émanant des établissements d'enseignement et de recherche français ou étrangers, des laboratoires publics ou privés. 


\title{
Two-level checkpointing and verifications for linear task graphs
}

\author{
Anne Benoit*, Aurélien Cavelan*, Yves Robert*†, Hongyang Sun* \\ ${ }^{*}$ Ecole Normale Supérieure de Lyon \& Inria, France \\ ${ }^{\dagger}$ University of Tennessee Knoxville, USA
}

\begin{abstract}
Fail-stop and silent errors are omnipresent on largescale platforms. Efficient resilience techniques must accommodate both error sources. To cope with the double challenge, a twolevel checkpointing and rollback recovery approach can be used, with additional verifications for silent error detection. A failstop error leads to the loss of the whole memory content, hence the obligation to checkpoint on a stable storage (e.g., an external disk). On the contrary, it is possible to use in-memory checkpoints for silent errors, which provide a much smaller checkpointing and recovery overhead. Furthermore, recent detectors offer partial verification mechanisms that are less costly than the guaranteed ones but do not detect all silent errors. In this paper, we show how to combine all of these techniques for HPC applications whose dependency graph forms a linear chain. We present a sophisticated dynamic programming algorithm that returns the optimal solution in polynomial time. Simulation results demonstrate that the combined use of multi-level checkpointing and verifications leads to improved performance compared to the standard single-level checkpointing algorithm.
\end{abstract}

Index Terms-resilience, fail-stop errors, silent errors, multilevel checkpoint, verification, dynamic programming.

\section{INTRODUCTION}

Resilience is one of the major challenges for extreme-scale computing [12], [13]. In particular, several types of errors should be considered. In addition to the classical fail-stop errors (such as hardware failures), silent errors, also known as silent data corruptions, constitute another threat that cannot be ignored any longer [19], [20], [24], [25]. In order to deal with both types of errors, a traditional checkpointing and rollback recovery strategy can be used [15], coupled with a verification mechanism to detect silent errors [8], [16], [22]. Such a verification mechanism can be either general-purpose (e.g., based on replication [17] or even triplication [18]) or application-specific (e.g., based on algorithm-based fault tolerance (ABFT) [11], on approximate re-execution for ODE and PDE solvers [9], or on orthogonality checks for Krylovbased sparse solvers [16], [22]).

Because verification mechanisms can be costly, alternative techniques capable of rapidly detecting silent errors, with the risk of missing some errors, have been recently developed and studied [2], [3], [10], [14]. We call these verifications partial verifications, while perfect verifications (with no error missed) are referred to as guaranteed verifications. Furthermore, rather than checkpointing only on a stable storage (e.g., an external disk), a lightweight mechanism of in-memory checkpoints can be provided: one keeps a local copy of the data that has not been corrupted when a silent error strikes, and it can be used to perform a recovery rapidly. However, such local copies are lost if a fail-stop error occurs, and hence copies on stable storage must also be provided.

Designing resilience algorithms by combining all of these techniques is challenging. In this paper, we deal with a simplified, yet realistic, application framework, where a set of application workflows exchange data at the end of their execution. Such a framework can be modeled as a task graph whose dependencies form a linear chain. This scenario corresponds to an HPC application whose workflow is partitioned into a succession of (typically large) tightly-coupled computational kernels, each of which is identified as a task. At the end of each task, we can perform either a partial verification or a guaranteed verification of the task output; or, probably less frequently, we can perform a guaranteed verification followed by a memory checkpoint (we do not take the risk of storing a corrupted checkpoint, hence the need for a guaranteed verification); or again, probably even less frequently, we can perform a guaranteed verification, a memory checkpoint and a disk checkpoint in a row.

The main contribution of this paper is a sophisticated dynamic programming algorithm that returns the optimal solution, i.e., the solution that minimizes the expected execution time, in polynomial time. The originality is that we combine both types of verifications and both types of checkpoints. Furthermore, we present extensive simulations that demonstrate the usefulness of mixing these techniques, and, in particular, we demonstrate the gain obtained thanks to multilevel checkpointing.

To the best of our knowledge, the interplay of verification mechanisms with two types of checkpoints, in-memory and disk-based, has never been investigated for task graphs. Our previous work [6] considers linear chains with a single checkpoint type and guaranteed verification (for the record, the pioneering work [23] for linear chains only deals with a single checkpoint type and no verification). The closest work to this paper is our recent work [7] for divisible load applications, where we consider the same combined framework (with two error sources, two checkpoint types and two verification mechanisms); however, in [7], we target longlasting executions, which are partitioned into periodic patterns that repeat over time, and we compute the best pattern up to first-order approximations. Here, we do not have the flexibility of divisible load applications, since we can insert resilience mechanisms only at the end of the execution of a task. We may 
well have a limited number of tasks, which prevents the use of any periodic strategy. Instead, we take a completely different approach and design (quite involved) dynamic programming algorithms that provide the optimal solution for any linear task graph.

The rest of this paper is organized as follows. We detail the model in Section II, before giving the dynamic programming algorithm in Section III. The simulation results are presented in Section IV. Finally, we conclude the paper in Section V with hints for future direction.

\section{MODEL}

We consider a linear chain of tasks $T_{1} \rightarrow T_{2} \rightarrow \cdots \rightarrow T_{n}$, where each task $T_{i}$ has a weight $w_{i}$ corresponding to the computational load. For notational convenience, we define $W_{i, j}=\sum_{k=i+1}^{j} w_{k}$ to be the time to execute tasks $T_{i+1}$ to $T_{j}$ for any $i<j$. Furthermore, we assume that hardware faults (fail-stop errors) and silent data corruptions (silent errors) coexist, as motivated in Section I. Since these two types of errors are caused by different sources, we assume that they are independent and that both occurrences follow Poisson process with arrival rates $\lambda_{f}$ and $\lambda_{s}$, respectively. The probability of having at least a fail-stop error during the execution of tasks $T_{i+1}$ to $T_{j}$ is given by $p_{i, j}^{f}=1-e^{-\lambda_{f} W_{i, j}}$ and that of having at least a silent error during the same execution is $p_{i, j}^{s}=1-e^{-\lambda_{s} W_{i, j}}$

To deal with both fail-stop and silent errors, resilience is provided through the use of a two-level checkpointing scheme coupled with an error detection (or verification) mechanism. When a fail-stop error strikes, the computation is interrupted immediately due to a hardware fault, so all the memory content is destroyed: we then recover from the last disk checkpoint or start again at the beginning of the application. On the contrary, when a silent error is detected, either by a partial verification or by a guaranteed one, we roll back to the nearest memory checkpoint, and recover from the memory copy there, which is much cheaper than recovering from the disk checkpoint.

We enforce that a memory checkpoint is always taken immediately before each disk checkpoint. This can be done with little overhead and it has been enforced in some practical multi-level checkpointing systems [5]. Also, a guaranteed verification is always taken immediately before each memory checkpoint, so that all checkpoints are valid (both memory and disk checkpoints), and hence only one memory checkpoint and one disk checkpoint need to be maintained at any time during the execution of the application. Furthermore, we assume that errors only strike the computations, while verifications, memory copies, and I/O transfers are protected from failures.

Let $C_{D}$ denote the cost of disk checkpointing, $C_{M}$ the cost of memory checkpointing, $R_{D}$ the cost of disk recovery, and $R_{M}$ the cost of memory recovery. Recall that when a disk recovery is done, we also need to restore the memory state. For simplicity, we assume that the cost $R_{M}$ is included in the cost $R_{D}$. Also, let $V^{*}$ denote the cost of guaranteed verification and $V$ the cost of a partial verification. The partial verification is also characterized by its recall, which is denoted by $r$ and represents the proportion of detected errors over all silent errors that have occurred during the execution. For notational convenience, we define $g=1-r$ to be the proportion of undetected errors. Note that the guaranteed verification can be considered as one with recall $r^{*}=1$. Since a partial verification usually incurs a much smaller cost and yet has a reasonable recall [3], [10], it is highly attractive for detecting silent errors, and we make use of them between guaranteed verifications.

The objective is to determine where to place disk checkpoints, memory checkpoints, guaranteed verifications and partial verifications, in order to minimize the expected execution time (or makespan) of the application.

\section{DYNAMIC PROGRAMMING}

In this section, we present a sophisticated multi-level dynamic programming algorithm to decide which tasks to verify, which tasks to checkpoint, as well as which type of verification or checkpoint to perform. Recall that we assumed a memory checkpoint always comes with a guaranteed verification to ensure the correctness of the results, and that a disk checkpoint always comes with a memory checkpoint, as motivated in Section II.

For convenience, we add a virtual task $T_{0}$, which is checkpointed on disk (and hence in memory), and whose recovery cost is zero. This accounts for the fact that it is always possible to restart the application from scratch at no extra cost.

We first describe in Section III-A a dynamic programming algorithm for the case where we use only guaranteed verifications, memory checkpoints and disk checkpoints. We then show how to extend this dynamic programming algorithm to include partial verifications in Section III-B.

\section{A. Without partial verifications}

Figures 1, 2, and 3 illustrate the idea of the algorithm without using partial verifications. The algorithm contains three dynamic programming levels, which are responsible for placing disk checkpoints (Figure 1), memory checkpoints (Figure 2), and guaranteed verifications (Figure 3), respectively. An additional step follows to compute the expected execution time between any two verifications. The following describes each step of the algorithm in detail.

Placing disk checkpoints. The first level focuses on placing disk checkpoints (see Figure 1). Let the function $E_{\text {disk }}\left(d_{2}\right)$ denote the expected time needed to successfully execute all the tasks from $T_{1}$ to $T_{d_{2}}$, where task $T_{d_{2}}$ is verified and checkpointed both on disk and in memory.

In this function, we try all possible locations for the last checkpoint before $T_{d_{2}}$. For each possible location $d_{1}$, we call the function recursively on $d_{1}$ (to place disk checkpoints before $T_{d_{1}}$ ), and we add the expected time needed to execute the tasks from $T_{d_{1}+1}$ to $T_{d_{2}}$. This is done through the $E_{\text {mem }}\left(d_{1}, d_{2}\right)$ function, which also decides where to place memory checkpoints, and accounts for the cost of memory checkpoints. The cost of the disk checkpoint $C_{D}$ is finally added after $T_{d_{2}}$. Note that a location $d_{1}=0$ means that no 


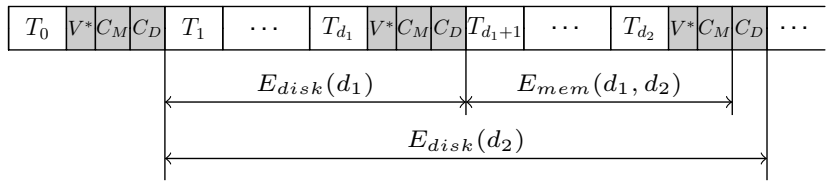

Figure 1. Placing disk checkpoints.

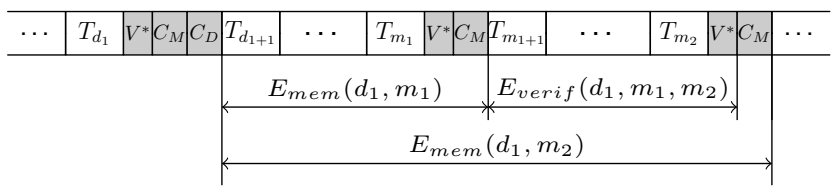

Figure 2. Placing memory checkpoints.

further disk checkpoints are added. In this case, we simply let $E_{\text {disk }}(0)=0$, which initializes the dynamic program. We can express $E_{d i s k}\left(d_{2}\right)$ as follows:

$$
E_{\text {disk }}\left(d_{2}\right)=\min _{0 \leq d_{1}<d_{2}}\left\{E_{\text {disk }}\left(d_{1}\right)+E_{\text {mem }}\left(d_{1}, d_{2}\right)+C_{D}\right\} .
$$

The total expected time needed to execute all the tasks $T_{1}$ to $T_{n}$ is given by $E_{d i s k}(n)$.

Placing memory checkpoints. The second level aims at placing additional memory checkpoints between two disk checkpoints (see Figure 2). The function is first called from the first level between two disk checkpoints, each of which also comes with a memory checkpoint. We define $E_{\text {mem }}\left(d_{1}, m_{2}\right)$ as the expected time needed for successfully executing all the tasks from $T_{d_{1}+1}$ to $T_{m_{2}}$, where there is a disk checkpoint at the end of task $T_{d_{1}}$, a memory checkpoint at the end of task $T_{m_{2}}$, and no other disk checkpoints. Note that there might be a disk checkpoint after $T_{m_{2}}$, for instance when we first call this function, but we do not account for the cost of this disk checkpoint in $E_{m e m}$, only for the cost of the memory checkpoint (the cost of the disk checkpoint is already accounted for in $E_{d i s k}$ ).

As before, we try all possible locations for the last memory checkpoint between tasks $T_{d_{1}}$ and $T_{m_{2}}$. For each possible location $m_{1}$, we call the function recursively on tasks $T_{d_{1}}$ to $T_{m_{1}}$, and then call the function for the next level, $E_{\text {verif }}\left(d_{1}, m_{1}, m_{2}\right)$, which computes the expected time needed to execute the tasks from $T_{m_{1}+1}$ to $T_{m_{2}}$ (and decides where to place verifications). Finally, we add the cost of the memory checkpoint $C_{M}$ following $T_{m_{2}}$. We can express $E_{m e m}\left(d_{1}, m_{2}\right)$ as follows:

$$
\begin{aligned}
& E_{\text {mem }}\left(d_{1}, m_{2}\right)= \\
& \min _{d_{1} \leq m_{1}<m_{2}}\left\{E_{\text {mem }}\left(d_{1}, m_{1}\right)+E_{\text {verif }}\left(d_{1}, m_{1}, m_{2}\right)+C_{M}\right\} .
\end{aligned}
$$

If $m_{1}=d_{1}$, there is no extra memory checkpoint between $d_{1}$ and $m_{2}$, and therefore we initialize the dynamic program with $E_{m e m}\left(d_{1}, d_{1}\right)=0$.

Placing additional verifications. The third level looks for where to insert additional verifications between two tasks with memory checkpoints (see Figure 3). The function is first called

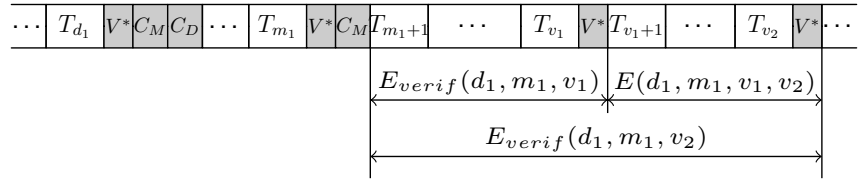

Figure 3. Placing verifications.

from the second level between two memory checkpoints, each of which also comes with a verification. Therefore, we define $E_{\text {verif }}\left(d_{1}, m_{1}, v_{2}\right)$ as the expected time needed for successfully executing all the tasks from $T_{m_{1}+1}$ to $T_{v_{2}}$, knowing that the last memory checkpoint is after $T_{m_{1}}$, the last disk checkpoint is after $T_{d_{1}}$, and there are no checkpoints between $T_{m_{1}+1}$ and $T_{v_{2}}$. Note that $E_{\text {verif }}\left(d_{1}, m_{1}, v_{2}\right)$ accounts only for the time required to execute and verify these tasks.

As before, we try all possible locations for the last verification between $T_{m_{1}}$ and $T_{v_{2}}$, and for each possible location $v_{1}$, we call the function recursively on tasks $T_{m_{1}}$ to $T_{v_{1}}$. Furthermore, we add the expected time needed to successfully execute the tasks $T_{v_{1}+1}$ to $T_{v_{2}}$, denoted by $E\left(d_{1}, m_{1}, v_{1}, v_{2}\right)$, knowing the position of the last disk checkpoint $d_{1}$ and the position of the last memory checkpoint $m_{1}$. We express $E_{\text {verif }}\left(d_{1}, m_{1}, v_{2}\right)$ as follows:

$$
\begin{aligned}
& E_{\text {verif }}\left(d_{1}, m_{1}, v_{2}\right)= \\
& \quad \min _{m_{1} \leq v_{1}<v_{2}}\left\{E_{\text {verif }}\left(d_{1}, m_{1}, v_{1}\right)+E\left(d_{1}, m_{1}, v_{1}, v_{2}\right)\right\} .
\end{aligned}
$$

Again, the case $v_{1}=m_{1}$ means that no further verifications are added, so we initialize the dynamic program with $E_{\text {verif }}\left(d_{1}, m_{1}, m_{1}\right)=0$. The verification cost at the end of $T_{v_{2}}$ is accounted for in the function $E\left(d_{1}, m_{1}, v_{1}, v_{2}\right)$.

Computing the expected execution time between two verifications. Finally, to compute the expected time needed for successfully executing several tasks between two verifications, we need the position of the last disk checkpoint $d_{1}$, the position of the last memory checkpoint $m_{1}$, and the positions of the two verifications $v_{1}$ and $v_{2}$.

On the one hand, if a fail-stop error occurs with probability $p_{v_{1}, v_{2}}^{f}$, then the execution stops and we must recover from the last disk checkpoint. In this case, we lose $T_{v_{1}, v_{2}}^{\text {lost }}$ time, pay the cost of recovery $R_{D}$ (set to 0 if $d_{1}=0$ ), and re-execute the tasks starting from $T_{d_{1}}$. The re-execution is done in three steps. First, we call $E_{m e m}\left(d_{1}, m_{1}\right)$ to compute the expected time needed to re-execute the tasks from the last disk checkpoint after $T_{d_{1}}$ to the last memory checkpoint after $T_{m_{1}}$. Then, we call the function $E_{\text {verif }}\left(d_{1}, m_{1}, v_{1}\right)$ to account for the time needed to re-execute the tasks between the last memory checkpoint after $T_{m_{1}}$ to the next verification after $T_{v_{1}}$. Finally, we re-execute tasks $T_{v_{1}+1}$ to $T_{v_{2}}$ with $E\left(d_{1}, m_{1}, v_{1}, v_{2}\right)$.

On the other hand, with probability $1-p_{v_{1}, v_{2}}^{f}$, there is no fail-stop error. In this case, we pay $W_{v_{1}, v_{2}}$ by executing all the tasks from $T_{v_{1}+1}$ to the next verification after $T_{v_{2}}$. Then we add the cost of the guaranteed verification $V^{*}$. After the verification, there is a probability $p_{v_{1}, v_{2}}^{s}$ of detecting a silent error, in which case we recover from the last memory 
checkpoint with a cost $R_{M}$ (set to 0 if $m_{1}=0$ ) and only re-execute the tasks from there by calling the function $E_{\text {verif }}\left(d_{1}, m_{1}, v_{1}\right)$ followed by $E\left(d_{1}, m_{1}, v_{1}, v_{2}\right)$ as before. Therefore, we have:

$$
\begin{aligned}
& E\left(d_{1}, m_{1}, v_{1}, v_{2}\right)= \\
& p_{v_{1}, v_{2}}^{f}\left(T_{v_{1}, v_{2}}^{\text {lost }}+R_{D}+E_{\text {mem }}\left(d_{1}, m_{1}\right)\right. \\
& \left.\quad+E_{\text {verif }}\left(d_{1}, m_{1}, v_{1}\right)+E\left(d_{1}, m_{1}, v_{1}, v_{2}\right)\right) \\
& \quad+\left(1-p_{v_{1}, v_{2}}^{f}\right)\left(W_{v_{1}, v_{2}}+V^{*}+p_{v_{1}, v_{2}}^{s}\left(R_{M}\right.\right. \\
& \left.\left.\quad+E_{\text {verif }}\left(d_{1}, m_{1}, v_{1}\right)+E\left(d_{1}, m_{1}, v_{1}, v_{2}\right)\right)\right) .
\end{aligned}
$$

In order to compute the expected execution time, we need to compute $T_{v_{1}, v_{2}}^{\text {lost }}$, which is the expected time loss due to a fail-stop error occurring during the execution of tasks $T_{v_{1}+1}$ to $T_{v_{2}}$. We can derive:

$$
\begin{aligned}
T_{v_{1}, v_{2}}^{\text {lost }} & =\int_{0}^{\infty} x \mathbb{P}\left(X=x \mid X<W_{v_{1}, v_{2}}\right) d x \\
& =\frac{1}{\mathbb{P}\left(X<W_{v_{1}, v_{2}}\right)} \int_{0}^{W_{v_{1}, v_{2}}} x \mathbb{P}(X=x) d x,
\end{aligned}
$$

where $\mathbb{P}(X=x)$ denotes the probability that a fail-stop error strikes at time $x$. By definition, we have $\mathbb{P}(X=x)=\lambda_{f} e^{-\lambda_{f} x}$ and $\mathbb{P}\left(X<W_{v_{1}, v_{2}}\right)=1-e^{-\lambda_{f} W_{v_{1}, v_{2}}}$. Integrating by parts, we get:

$$
T_{v_{1}, v_{2}}^{\text {lost }}=\frac{1}{\lambda_{f}}-\frac{W_{v_{1}, v_{2}}}{e^{\lambda_{f} W_{v_{1}, v_{2}}-1}} .
$$

Now, substituting $T_{v_{1}, v_{2}}^{\text {lost }}$ into Equation (2) and simplifying, we obtain:

$$
\begin{aligned}
& E\left(d_{1}, m_{1}, v_{1}, v_{2}\right)=e^{\lambda_{s} W_{v_{1}, v_{2}}}\left(\frac{e^{\lambda_{f} W_{v_{1}, v_{2}}}-1}{\lambda_{f}}+V^{*}\right) \\
& \quad+e^{\lambda_{s} W_{v_{1}, v_{2}}}\left(e^{\lambda_{f} W_{v_{1}, v_{2}}}-1\right)\left(R_{D}+E_{m e m}\left(d_{1}, m_{1}\right)\right) \\
& \quad+\left(e^{\left(\lambda_{s}+\lambda_{f}\right) W_{v_{1}, v_{2}}}-1\right) E_{\text {verif }}\left(d_{1}, m_{1}, v_{1}\right) \\
& \quad+\left(e^{\lambda_{s} W_{v_{1}, v_{2}}}-1\right) R_{M} .
\end{aligned}
$$

Complexity. The complexity is dominated by the computation of the table $E_{\text {verif }}\left(d_{1}, m_{1}, v_{2}\right)$, which contains $O\left(n^{3}\right)$ entries, and each entry depends on at most $n$ other entries that are already computed. All tables are computed in a bottom-up fashion, from the left to the right of the intervals. Hence, the overall complexity of the algorithm is $O\left(n^{4}\right)$.

\section{B. With partial verifications}

It may be beneficial to further add partial verifications between two guaranteed verifications. The intuitive idea would be to add yet another level to the dynamic programming algorithm, and to replace $E\left(d_{1}, m_{1}, v_{1}, v_{2}\right)$ in Equation (1) by a call to a function $E_{\text {partial }}^{(\text {intuitive }}\left(d_{1}, m_{1}, v_{1}, p_{2}, v_{2}\right)$, with $p_{2}=v_{2}$, which would compute the expected time needed to execute all the tasks from $T_{v_{1}+1}$ to $T_{p_{2}}$ and add further partial verifications (computed from the left to the right).
However, while the dynamic programming approach was rather intuitive without partial verifications, the problem becomes much harder with partial verifications. The main reason is that when computing an interval between two partial verifications, there is a probability $g$ that the error remains undetected after the partial verification. When this happens, we need to account for the time lost executing the following tasks until the error is eventually detected (by the subsequent partial verifications or in the worst case by the guaranteed verification) or until the execution is interrupted by a failstop error. This is only possible if we know the optimal positions of the partial verifications after the interval up to the next guaranteed verification. This requires the dynamic programming algorithm to first compute the values at the right of the current interval, hence progressing the opposite way as what was done so far. Therefore, the function becomes $E_{\text {partial }}\left(d_{1}, m_{1}, v_{1}, p_{1}, v_{2}\right)$ (expected time needed to execute all the tasks from $T_{p_{1}+1}$ to $T_{v_{2}}$ ), and it tries all positions $p_{2}$ for the next partial verification. But then, it also requires to remove some terms that account for re-executed work from the intervals on the left of the current interval (because we do not have this information yet), and to re-inject them later in the computation. In the end, we have quite a complicated algorithm.

Expected lost time in case of silent error. First, we compute $E_{\text {right }}\left(d_{1}, m_{1}, v_{1}, p_{1}, v_{2}\right)$, the expected time lost executing the tasks $T_{p_{1}+1}$ to $T_{v_{2}}$, assuming that there was a silent error in this interval. This computation uses $p_{2}$, the optimal position of the verification immediately following $p_{1}$, which is computed with the dynamic programming. Indeed, $T_{p_{2}}$ may detect the error or not. If the error is detected by $T_{p_{2}}$, we lose $W_{p_{1}, p_{2}}+V+$ $R_{M}$ work, while we use $E_{\text {right }}\left(d_{1}, m_{1}, v_{1}, p_{2}, v_{2}\right)$ if the error remains undetected. Also, we consider fail-stop errors only between $T_{p_{1}+1}$ and $T_{p_{2}}$, because fail-stop errors between $T_{p_{2}+1}$ and $T_{v_{2}}$ will be accounted for in $E_{\text {right }}\left(d_{1}, m_{1}, v_{1}, p_{2}, v_{2}\right)$. Note that even if we know that there is a silent error in the interval, we may need to recover from a fail-stop error if it strikes before the silent error is detected. Altogether, we have:

$$
\begin{aligned}
& E_{\text {right }}\left(d_{1}, m_{1}, v_{1}, p_{1}, v_{2}\right)= \\
& p_{p_{1}, p_{2}}^{f}\left(T_{p_{1}, p_{2}}^{\text {lost }}+R_{D}+E_{m e m}\left(d_{1}, m_{1}\right)\right) \\
& +\left(1-p_{p_{1}, p_{2}}^{f}\right)\left(W_{p_{1}, p_{2}}+V+(1-g) R_{M}\right. \\
& \left.\quad+g E_{\text {right }}\left(d_{1}, m_{1}, v_{1}, p_{2}, v_{2}\right)\right) \\
& =\left(1-e^{-\lambda_{f} W_{p_{1}, p_{2}}}\right)\left(\frac{1}{\lambda_{f}}-\frac{W_{p_{1}, p_{2}}}{e^{\lambda_{f} W_{p_{1}, p_{2}}-1}}\right. \\
& \left.\quad+R_{D}+E_{m e m}\left(d_{1}, m_{1}\right)\right) \\
& +e^{-\lambda_{f} W_{p_{1}, p_{2}}\left(W_{p_{1}, p_{2}}+V+(1-g) R_{M}\right.} \\
& \left.\quad+g E_{r i g h t}\left(d_{1}, m_{1}, v_{1}, p_{2}, v_{2}\right)\right) .
\end{aligned}
$$

The initialization is $E_{\text {right }}\left(d_{1}, m_{1}, v_{1}, v_{2}, v_{2}\right)=R_{M}$. Indeed, in this case, there is no task to execute, and if there was a silent error, it is therefore immediately detected by $v_{2}$ (a guaranteed 


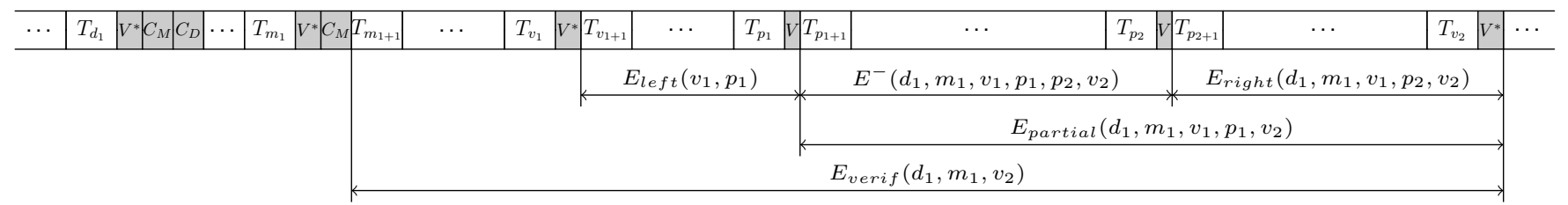

Figure 4. Placing partial verifications.

verification), and we just pay $R_{M}$. Knowing $p_{2}$, we are therefore able to compute all values of $E_{\text {right }}$. We will see later how we use this knowledge in $E_{\text {right }}$. Note that the time to reexecute the tasks after a recovery is omitted here, since it will be accounted for when computing $E\left(d_{1}, m_{1}, v_{1}, p_{1}, p_{2}, v_{2}\right)$, the expected time needed to successfully execute all the tasks between two partial verifications (from $T_{p_{1}+1}$ to $T_{p_{2}}$ ).

Expected time to compute tasks $T_{p_{1}+1}$ to $T_{p_{2}}$. Figure 4 shows all the tasks involved in the computation of an interval consisting of several tasks between two partial verifications at $p_{1}$ and $p_{2}$. Let $E\left(d_{1}, m_{1}, v_{1}, p_{1}, p_{2}, v_{2}\right)$ denote the expected time needed to successfully execute all the tasks from $T_{p_{1}+1}$ to $T_{p_{2}}$, knowing that the last disk checkpoint is after $T_{d_{1}}$, the last memory checkpoint is after $T_{m_{1}}$, the last guaranteed verification is after $T_{v_{1}}$, and the next guaranteed verification is after $T_{v_{2}}$.

On the one hand, if a fail-stop error occurs with probability $p_{p_{1}, p_{2}}^{f}$, then the task stops and we must recover from the last disk checkpoint. We lose $T_{p_{1}, p_{2}}^{\text {lost }}$ time, we pay the cost for the disk recovery $R_{D}$, and we need to re-execute the tasks starting from $T_{d_{1}}$. This is done in three steps: first we call $E_{m e m}\left(d_{1}, m_{1}\right)$ to compute the expected time needed to re-execute the tasks from the last disk checkpoint after $T_{d_{1}}$ to the last memory checkpoint after $T_{m_{1}}$. Then we call the function $E_{\text {verif }}\left(d_{1}, m_{1}, v_{1}\right)$ to account for the time needed to re-execute the tasks between the last memory checkpoint after $T_{m_{1}}$ to the next guaranteed verification after $T_{v_{1}}$, and finally we are left with the remaining tasks between $T_{v_{1}+1}$ and $T_{p_{1}}$. Let $E_{\text {left }}\left(v_{1}, p_{1}\right)$ denote the expected time needed to re-execute all the tasks from $T_{v_{1}+1}$ to $T_{p_{1}}$. Finally, we can reexecute tasks $T_{v_{1}+1}$ to $T_{v_{2}}$ by calling $E\left(d_{1}, m_{1}, v_{1}, p_{1}, p_{2}, v_{2}\right)$.

On the other hand, there is a probability $\left(1-p_{p_{1}, p_{2}}^{f}\right)$ of having no fail-stop errors. In that case, we execute all the tasks from $T_{p_{1}+1}$ to the next verification after $T_{p_{2}}$ and we pay $W_{p_{1}, p_{2}}$. Then we add the cost $V$ for the verification. After the partial verification, there is a probability $p_{p_{1}, p_{2}}^{s}$ of having a silent error. In this case, we pay a recovery from the last memory checkpoint $\left(R_{M}\right)$ and re-executed the tasks from there: we call $E_{\text {verif }}\left(d_{1}, m_{1}, v_{1}\right)$, followed by $E_{l e f t}\left(v_{1}, p_{1}\right)$ and $E\left(d_{1}, m_{1}, v_{1}, p_{1}, p_{2}, v_{2}\right)$. Furthermore, if the error was not detected (with probability $g$ ), we use $E_{\text {right }}\left(d_{1}, m_{1}, v_{1}, p_{2}, v_{2}\right)$ to compute the expected time lost executing the tasks following $T_{p_{2}}$, knowing that there is an undetected silent error (as explained earlier). Therefore, we have:

$$
\begin{aligned}
& E\left(d_{1}, m_{1}, v_{1}, p_{1}, p_{2}, v_{2}\right)= \\
& p_{p_{1}, p_{2}}^{f}\left(T_{p_{1}, p_{2}}^{\text {lost }}+R_{D}+E_{m e m}\left(d_{1}, m_{1}\right)+E_{\text {verif }}\left(d_{1}, m_{1}, v_{1}\right)\right. \\
& \left.+E_{\text {left }}\left(v_{1}, p_{1}\right)+E\left(d_{1}, m_{1}, v_{1}, p_{1}, p_{2}, v_{2}\right)\right) \\
& +\left(1-p_{p_{1}, p_{2}}^{f}\right) \\
& \quad\left(W_{p_{1}, p_{2}}+V+p_{p_{1}, p_{2}}^{s}\left(E_{\text {verif }}\left(d_{1}, m_{1}, v_{1}\right)\right.\right. \\
& +E_{\text {left }}\left(v_{1}, p_{1}\right)+E\left(d_{1}, m_{1}, v_{1}, p_{1}, p_{2}, v_{2}\right) \\
& \left.\left.+(1-g) R_{M}+g E_{\text {right }}\left(d_{1}, m_{1}, v_{1}, p_{2}, v_{2}\right)\right)\right) .
\end{aligned}
$$

Substituting $T_{p_{1}, p_{2}}^{\text {lost }}$ into the equation above and simplifying, we obtain:

$$
\begin{aligned}
& E\left(d_{1}, m_{1}, v_{1}, p_{1}, p_{2}, v_{2}\right)=e^{\lambda_{s} W_{p_{1}, p_{2}}}\left(\frac{e^{\lambda_{f} W_{p_{1}, p_{2}}}-1}{\lambda_{f}}+V\right) \\
& +e^{\lambda_{s} W_{p_{1}, p_{2}}}\left(e^{\lambda_{f} W_{p_{1}}, p_{2}}-1\right)\left(R_{D}+E_{\text {mem }}\left(d_{1}, m_{1}\right)\right) \\
& +\left(e^{\left(\lambda_{s}+\lambda_{f}\right) W_{p_{1}, p_{2}}}-1\right)\left(E_{\text {verif }}\left(d_{1}, m_{1}, v_{1}\right)+E_{l e f t}\left(v_{1}, p_{1}\right)\right) \\
& +\left(e^{\lambda_{s} W_{p_{1}, p_{2}}}-1\right)\left((1-g) R_{M}+g E_{\text {right }}\left(d_{1}, m_{1}, v_{1}, p_{2}, v_{2}\right)\right) .
\end{aligned}
$$

Finally, because we do not know at this point how to compute $E_{\text {left }}\left(v_{1}, p_{1}\right)$, we remove the term

$$
\left(e^{\left(\lambda_{s}+\lambda_{f}\right) W_{p_{1}, p_{2}}}-1\right) E_{l e f t}\left(v_{1}, p_{1}\right)
$$

from $E\left(d_{1}, m_{1}, v_{1}, p_{1}, p_{2}, v_{2}\right)$. This corresponds to the amount of time needed to re-execute all the tasks from $T_{v_{1}+1}$ to $T_{p_{1}}$ when there is an error between $T_{p_{1}+1}$ and $T_{p_{2}}$. This time will be added back when computing $E_{\text {partial }}$, as explained below. Therefore, we introduce the modified expression of $E$, denoted $E^{-}$, as follows:

$$
\begin{aligned}
& E^{-}\left(d_{1}, m_{1}, v_{1}, p_{1}, p_{2}, v_{2}\right)=e^{\lambda_{s} W_{p_{1}, p_{2}}}\left(\frac{e^{\lambda_{f} W_{p_{1}, p_{2}}}-1}{\lambda_{f}}+V\right) \\
& +e^{\lambda_{s} W_{p_{1}, p_{2}}}\left(e^{\lambda_{f} W_{p_{1}, p_{2}}}-1\right)\left(R_{D}+E_{m e m}\left(d_{1}, m_{1}\right)\right) \\
& +\left(e^{\left(\lambda_{s}+\lambda_{f}\right) W_{p_{1}, p_{2}}}-1\right)\left(E_{\text {verif }}\left(d_{1}, m_{1}, v_{1}\right)\right) \\
& +\left(e^{\lambda_{s} W_{p_{1}, p_{2}}}-1\right)\left((1-g) R_{M}+g E_{\text {right }}\left(d_{1}, m_{1}, v_{1}, p_{2}, v_{2}\right)\right) .
\end{aligned}
$$

Computing $E_{\text {partial }}\left(d_{1}, m_{1}, v_{1}, p_{1}, v_{2}\right)$, the expected time needed to execute all the tasks from $T_{p_{1}+1}$ to $T_{v_{2}}$ (and placing extra partial verifications). Finally, we need to compute $E_{\text {partial }}$ and to decide when to use additional partial verifications on tasks that are not yet verified. The function is first called from the third level between two guaranteed verifications, and $p_{1}$ is originally set to $v_{1}$. Therefore, $E_{\text {partial }}\left(d_{1}, m_{1}, v_{1}, p_{1}, v_{2}\right)$ denotes the expected time needed 
to execute all the tasks from $T_{p_{1}+1}$ to $T_{v_{2}}$, where $T_{p_{1}}$ is followed by a partial verification (with the exception of the first call) and $T_{v_{2}}$ is followed by a guaranteed verification, knowing the position of the last disk checkpoint $d_{1}$, the position of the last memory checkpoint $m_{1}$ and the position of the last guaranteed verification $v_{1}$.

Contrarily to the expressions derived in Section III-A, note that partial verifications are placed from the left to the right. We use the expression of $E^{-}$, trying all possible positions $p_{2}$ for the partial verification following $p_{1}$, and we account for the fact that tasks between $T_{p_{1}+1}$ and $T_{p_{2}}$ may be re-executed several times (because we removed $E_{\text {left }}$ from $E^{-}$). In fact, for any number of partial verifications between $p_{2}$ and $v_{2}$, we can show that $E^{-}\left(d_{1}, m_{1}, v_{1}, p_{1}, p_{2}, v_{2}\right)$ is re-executed $e^{\left(\lambda_{s}+\lambda_{f}\right) W_{p_{2}, v_{2}}}$ times, and hence we obtain:

$$
\begin{aligned}
& E_{\text {partial }}\left(d_{1}, m_{1}, v_{1}, p_{1}, v_{2}\right)= \\
& \min _{p_{1}<p_{2} \leq v_{2}}\left\{E^{-}\left(d_{1}, m_{1}, v_{1}, p_{1}, p_{2}, v_{2}\right) \cdot e^{\left(\lambda_{s}+\lambda_{f}\right) W_{p_{2}, v_{2}}}\right. \\
& \left.\quad+E_{\text {partial }}\left(d_{1}, m_{1}, v_{1}, p_{2}, v_{2}\right)\right\}
\end{aligned}
$$

The initialization case is for $p_{2}=v_{2}$. Then, there is no re-execution of the interval induced by errors to the right of $p_{2}$ (within an $E_{\text {left }}$ ), and therefore we compute only once $E^{-}\left(d_{1}, m_{1}, v_{1}, p_{1}, v_{2}, v_{2}\right)$. Furthermore, the interval is ended by a guaranteed verification, and therefore we add the corresponding verification cost:

$$
\begin{aligned}
& E_{\text {partial }}\left(d_{1}, m_{1}, v_{1}, v_{2}, v_{2}\right)= \\
& \quad E^{-}\left(d_{1}, m_{1}, v_{1}, p_{1}, v_{2}, v_{2}\right)+e^{\left(\lambda_{s}+\lambda_{f}\right) W_{p_{1}, v_{2}}}\left(V^{*}-V\right) .
\end{aligned}
$$

Because partial verifications are placed from the left to the right, when implementing the algorithm, we first compute all values of $E_{\text {partial }}$ on the right of the interval, which are needed to progress towards the left. This is why we always have the values of the next $p_{2}$ when computing $E_{\text {right }}$, which correspond to the minimum value selected by $E_{\text {partial }}$. However, it was not possible to derive the values of the reexecution for the left part of the interval, hence the trick to compute the number of times each interval is re-executed, due to a failure on the right (the term $E_{\text {left }}$ that is removed from the initial expression of $E$ ).

Accounting for re-executions on the left. Finally, let us show that for any number of partial verifications between $p_{2}$ and $v_{2}$, the function $E^{-}\left(d_{1}, m_{1}, v_{1}, p_{1}, p_{2}, v_{2}\right)$ re-executes an $e^{\left(\lambda_{s}+\lambda_{f}\right) W_{p_{2}, v_{2}}}$ amount of work. If there are no partial verifications after $p_{2}$, then it is executed once when progressing within the computation, and we also need to account for the $e^{\left(\lambda_{s}+\lambda_{f}\right) W_{p_{2}, v_{2}}}-1$ re-executed work due to the term $E_{l e f t}$ that was suppressed from $E^{-}\left(d_{1}, m_{1}, v_{1}, p_{2}, v_{2}, v_{2}\right)$.

With one intermediate partial verification $p_{3}$ between $p_{2}$ and $v_{2}$, the same reasoning shows that there is an amount of $e^{\left(\lambda_{s}+\lambda_{f}\right) W_{p_{2}, p_{3}}}$ re-executed work coming from the initial execution and the $E_{\text {left }}$ term suppressed from $E^{-}\left(d_{1}, m_{1}, v_{1}, p_{2}, p_{3}, v_{2}\right)$. Furthermore, there is an amount of $\left(e^{\left(\lambda_{s}+\lambda_{f}\right) W_{p_{3}, v_{2}}}-1\right)$ re-executed work coming from the
$E_{\text {left }}$ term of $E^{-}\left(d_{1}, m_{1}, v_{1}, p_{3}, v_{2}, v_{2}\right)$. In turn, this reexecuted work incurs $e^{\left(\lambda_{s}+\lambda_{f}\right) W_{p_{2}, p_{3}}}$ re-executed work (initial execution and re-executions due to the $E_{\text {left }}$ term coming from $\left.E^{-}\left(d_{1}, m_{1}, v_{1}, p_{2}, p_{3}, v_{2}\right)\right)$. Overall, the number of reexecuted work is finally

$$
e^{\left(\lambda_{s}+\lambda_{f}\right) W_{p_{2}, p_{3}}}+\left(e^{\left(\lambda_{s}+\lambda_{f}\right) W_{p_{2}, p_{3}}}\right)\left(e^{\left(\lambda_{s}+\lambda_{f}\right) W_{p_{3}, v_{2}}}-1\right),
$$

and therefore there is a total amount of $e^{\left(\lambda_{s}+\lambda_{f}\right) W_{p_{2}, v_{2}}}$ reexecuted work. It is easy to extend this reasoning to any number of intervals by induction, assuming that it is true for $i$ intermediate partial verifications $p v_{i}, \ldots, p v_{1}$, and adding a partial verification $p v_{i+1}$ between $p_{2}$ and $p v_{i}$. The same reasoning holds.

Complexity. Clearly, the complexity is now dominated by the computation of the table $E_{\text {partial }}\left(d_{1}, m_{1}, v_{1}, p_{1}, v_{2}\right)$, which contains $O\left(n^{5}\right)$ entries, and each entry depends on at most $n$ other entries that are already computed. Hence, the overall complexity of the algorithm is $O\left(n^{6}\right)$.

\section{Performance evaluation}

In this section, we conduct a set of simulations to assess the relative efficiency of our approach under realistic scenarios. We instantiate the model with actual parameters from the literature and we compare the performance of three algorithms: (i) a single-level algorithm $\mathrm{A}_{D V^{*}}$ with only disk checkpoints (and additional guaranteed verifications), (ii) a two-level algorithm combining memory and disk checkpoints $\mathrm{A}_{D M V^{*}}$ (as in Section III-A), and (iii) the complete algorithm using additional partial verifications $\mathrm{A}_{D M V}$ (as in Section III-B). The optimal positions of verifications and disk checkpoints can be easily derived for $\mathrm{A}_{D V^{*}}$, using a simplification of the proposed dynamic programming algorithm in Section III-A with no additional memory checkpoints.

Simulation setup. We make several assumptions on the input parameters. First, we assume that recovery costs and checkpoint costs are similar; following [19], [21], we set recovery costs to be the same as checkpoint costs, i.e., $R_{D}=C_{D}$ and $R_{M}=C_{M}$. Then, we assume that a guaranteed verification must check all the data in memory, making its cost in the same order as that of a memory checkpoint, i.e., $V^{*}=C_{M}$. Furthermore, we assume partial verifications similar to those proposed in [3], [4], [10], with very low cost while offering good recalls. In the following, we set $V=V^{*} / 100$ and $r=0.8$. The total computational weight is set to be 25000 seconds and it is distributed uniformly among up to $n=50$ tasks in three different patterns shown as follows.

(1) Uniform: all tasks share the same weight $W / n$, as in matrix multiplication or in some iterative stencil kernels.

(2) Decrease: task $T_{i}$ has weight $\alpha(n+1-i)^{2}$, where $\alpha \approx$ $3 \mathrm{~W} / n^{3}$; this quadratically decreasing function resembles some dense matrix solvers, e.g., by using LU or QR factorization.

(3) HighLow: a set of tasks with large weight is followed by a set of tasks with small weight. In the simulations, we set $10 \%$ of the tasks to be large and let them contain $60 \%$ of the total computational weight. 
We point out that all these choices are somewhat arbitrary and can easily be modified in the evaluations; we believe they represent reasonable values for current and next-generation HPC applications. The code is publicly available at http: //graal.ens-lyon.fr/ $\sim$ yrobert/chain2levels.zip for the interested readers to experiment with their own parameters.

Platform settings. Table I presents the four platforms used in the simulations and their main parameters. These platforms have been used to evaluate the Scalable Checkpoint/Restart (SCR) library by Moody et al. [19], who provide accurate measurements for $\lambda_{f}, \lambda_{s}, C_{D}$ and $C_{M}$ using real applications. Note that the Hera platform has the worst error rates, with a platform MTBF of 12.2 days for fail-stop errors and 3.4 days for silent errors. In comparison, and despite its higher number of nodes, the Coastal platform features a platform MTBF of 28.8 days for fail-stop errors and 5.8 days for silent errors. In addition, the last platform uses SSD technology for memory checkpointing, which provides more data space, at the cost of higher checkpointing costs.

Table I

PLATFORM PARAMETERS.

\begin{tabular}{|c|c|c|c|c|c|}
\hline platform & \#nodes & $\lambda_{f}$ & $\lambda_{s}$ & $C_{D}$ & $C_{M}$ \\
\hline Hera & 256 & $9.46 \mathrm{e}-7$ & $3.38 \mathrm{e}-6$ & $300 s$ & $15.4 s$ \\
Atlas & 512 & $5.19 \mathrm{e}-7$ & $7.78 \mathrm{e}-6$ & $439 s$ & $9.1 s$ \\
Coastal & 1024 & $4.02 \mathrm{e}-7$ & $2.01 \mathrm{e}-6$ & $1051 s$ & $4.5 s$ \\
Coastal SSD & 1024 & $4.02 \mathrm{e}-7$ & $2.01 \mathrm{e}-6$ & $2500 \mathrm{~s}$ & $180.0 \mathrm{~s}$ \\
\hline
\end{tabular}

Impact of number of tasks. The first column of Figure 5 presents, for each platform, the normalized makespan with respect to the error-free execution time for different numbers of tasks with the Uniform weight distribution. Note that varying the number of tasks has an impact on both the size of the tasks and the maximum number of checkpoints and verifications an algorithm can place. When the number of tasks is small (e.g., less than 5), the probability of having an error during the execution (either a fail-stop or a silent) increases quickly (more than $10 \%$ on Hera) for a single task. As a result, the application experiences more recoveries and re-executions with larger tasks, which increases the execution overhead. However, when the number of tasks is large enough, the size of the tasks becomes small and the probability of having an error during the execution of one task drops significantly, reducing the recovery and re-execution costs at the same time.

Single-level algorithm $\mathbf{A}_{D V^{*}}$. The second column of Figure 5 shows the numbers of disk checkpoints (with associated memory checkpoints) and guaranteed verifications used by the $\mathrm{A}_{D V^{*}}$ algorithm on the four platforms and for different numbers of tasks. We observe that a large number of guaranteed verifications is placed by the algorithm while the number of checkpoints remains relatively small (i.e., less than 5 for all the platforms). This is because checkpoints are costly, and verifications help to reduce the amount of time lost due to silent errors. Since verifications are cheaper, the algorithm tends to place as many of them as possible, except when their relative costs also become high (e.g., on Coastal SSD).
Two-level algorithm $\mathbf{A}_{D M V^{*}}$. The third column of Figure 5 presents the numbers of disk checkpoints, memory checkpoints and guaranteed verifications used by the $\mathrm{A}_{D M V^{*}}$ algorithm on the four platforms and for different number of tasks. We observe that the number of guaranteed verifications remains similar to that placed by the $\mathrm{A}_{D V^{*}}$ algorithm. However, the two-level algorithm uses additional memory checkpoints, which drastically reduces the amount of time lost in reexecution when a silent error is detected. In particular, we observe that the algorithm $\mathrm{A}_{D M V^{*}}$ always leads to a better makespan compared to the single-level algorithm $\mathrm{A}_{D V^{*}}$, with an improvement of $2 \%$ on Hera and $5 \%$ on Atlas, as shown in the first column of Figure 5. This demonstrates the usefulness of the multi-level checkpointing approach.

Two-level algorithm with partial verifications $\mathbf{A}_{D M V}$. The last column of Figure 5 presents the numbers of disk checkpoints, memory checkpoints, guaranteed verifications and additional partial verifications used by the $\mathrm{A}_{D M V}$ algorithm on the four platforms and for different numbers of tasks. Although partial verifications are always more cost-effective than guaranteed ones, due to the imperfect recall, they are only worth it if one can use a lot of them, which is only possible when the number of tasks is large enough. Therefore, the algorithm only starts to use partial verifications when the number of tasks is greater than 30 on Hera, 40 on Coastal and 50 on Atlas, where the silent error rate is the highest among the four platforms. In our setting, adding partial verifications has a limited impact on the makespan, with the exception of the Coastal SSD platform, where the cost of checkpoints and verifications are much higher than on the other platforms. Partial verifications, being 100 times cheaper than guaranteed verifications, remain the only affordable resilience tool on this platform. In this case, we observe an improved makespan (around $1 \%$ with 50 tasks) compared to the $\mathrm{A}_{D M V^{*}}$ algorithm, as shown in the first column of Figure 5.

Distribution of checkpoints and verifications. Figure 6 shows the positions of the disk checkpoints, memory checkpoints, verifications and partial verifications obtained by running the $\mathrm{A}_{D M V}$ algorithm on each of the four platforms and for 50 tasks with the uniform distribution. For all platforms, the algorithm does not perform any additional disk checkpoints. These being costly, the algorithm rather uses more memory checkpoints and verifications. On most platforms, the optimal solution is a combination of equi-spaced memory checkpoints and guaranteed verifications, with additional partial verifications in-between. However, on the Coastal SSD platform, the cost of checkpoints and verifications is substantially higher, which leads the algorithm to choose partial verifications over guaranteed ones.

Decrease pattern. In the following, we focus on the platforms Hera and Coastal SSD, which represent both extremes in terms of size (number of processors) and hardware used for memory checkpointing (RAM and SSD, respectively). The first column of Figure 7 presents the performance of 

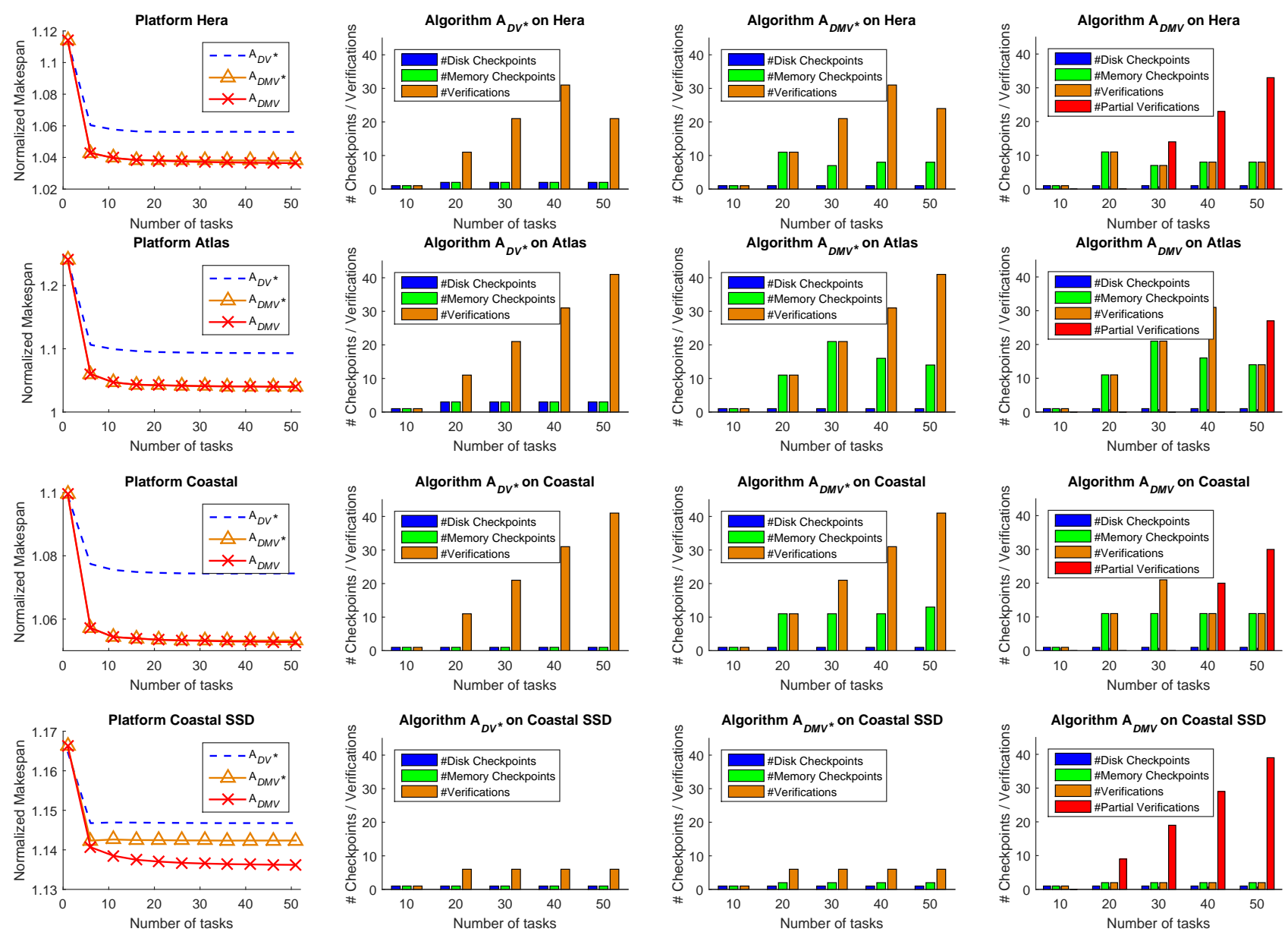

Figure 5. Performance of the three algorithms on each platform with the Uniform pattern. Each row corresponds to one platform.

Platform Hera with $\mathrm{A}_{D M V}$ and $\mathrm{n}=\mathbf{5 0}$

Disk ckpts

Memory ckpts

Guaranteed verifs

Partial verifs

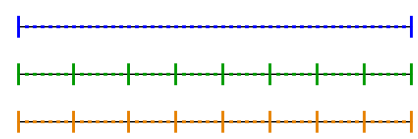

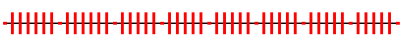

Platform Coastal with $\mathrm{A}_{D M V}$ and $\mathrm{n}=50$

Disk ckpts
Memory ckpts
Guaranteed verifs
Partial verifs

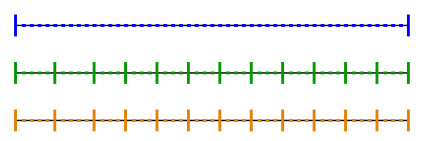

HH-HH-HHHHHHH-HHH-HHH-HHH
Platform Atlas with $\mathrm{A}_{D M V}$ and $\mathrm{n}=50$

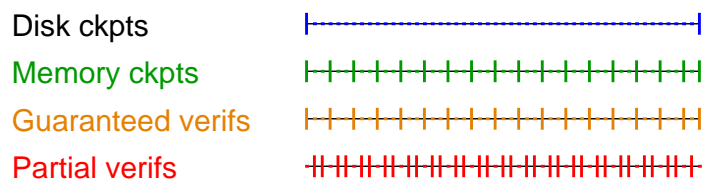

Platform Coastal SSD with $\mathrm{A}_{D M V}$ and $\mathrm{n}=50$

Disk ckpts
Memory ckpts
Guaranteed verifs
Partial verifs

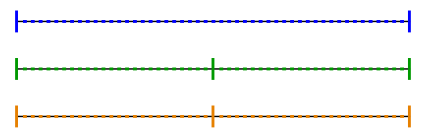

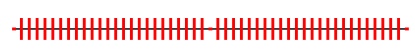

Figure 6. Distribution of disk checkpoints, memory checkpoints and verifications for the $\mathrm{A}_{D M V}$ algorithm on each platform with the Uniform pattern.

the three algorithms for different number of tasks and for the Decrease pattern. The second column shows the number of disk checkpoints, memory checkpoints, guaranteed and partial verifications given by the $\mathrm{A}_{D M V}$ algorithm. The third column is a visual representation of the corresponding solution obtained for 50 tasks and with the same configuration. We observe that the makespan obtained is very similar for all three algorithms (with a slight advantage for $\mathrm{A}_{D M V}$ ). Since 

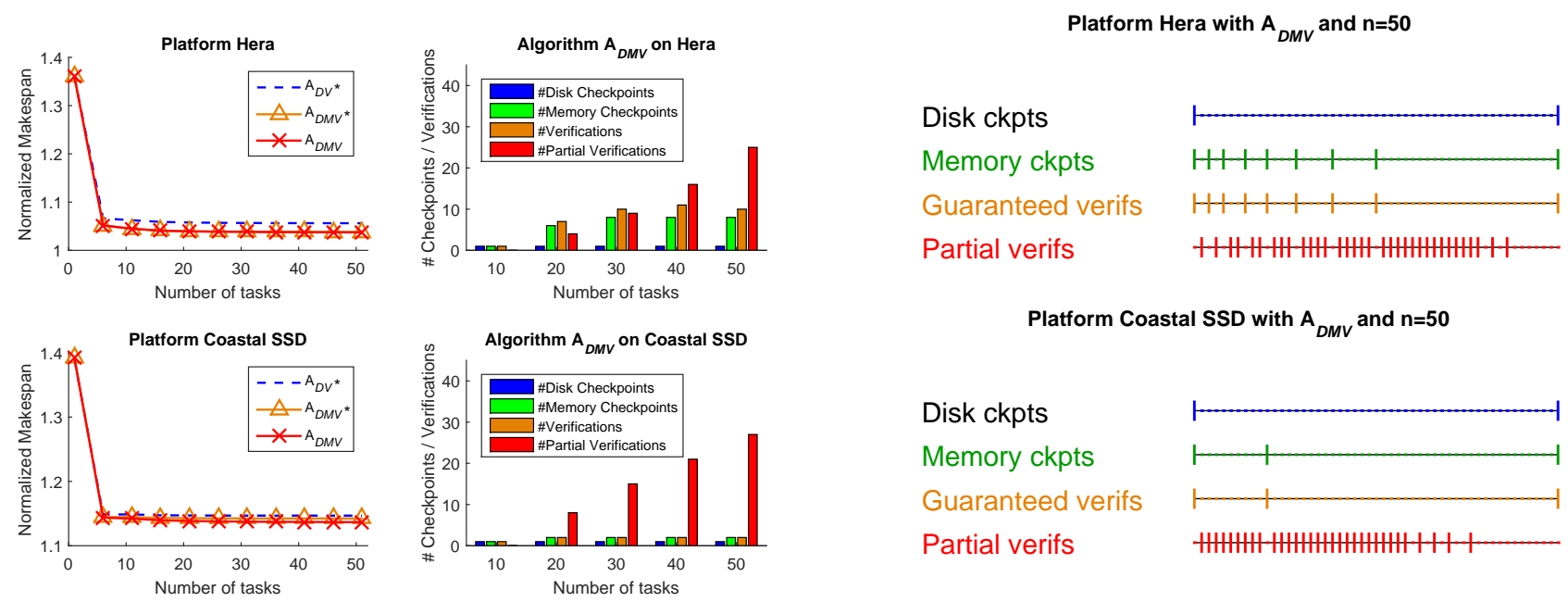

Platform Coastal SSD with $\mathrm{A}_{D M V}$ and $\mathrm{n}=50$

Figure 7. Performance of the three algorithms, and distribution of disk checkpoints, memory checkpoints and verifications (for the $\mathrm{A}_{D M V}$ algorithm) on platforms Hera and Coastal SSD with the Decrease pattern.
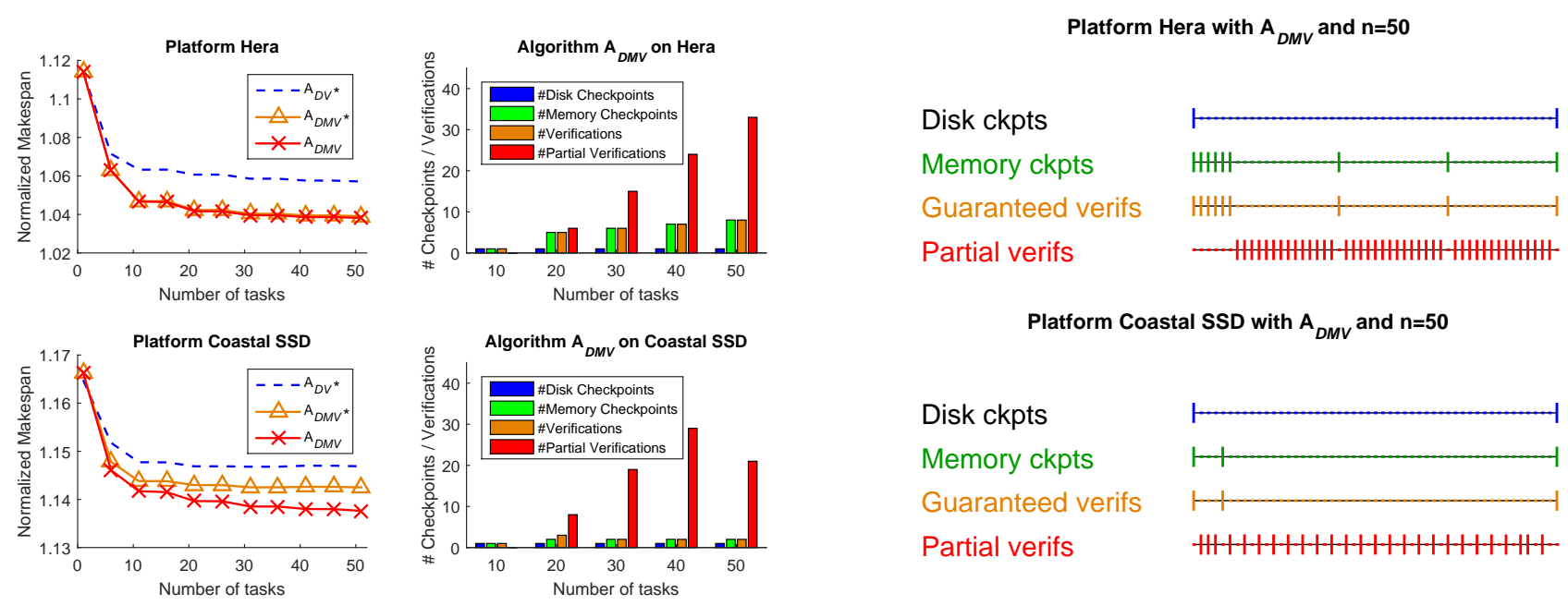

Disk ckpts
Memory ckpts
Guaranteed verifs
Partial verifs

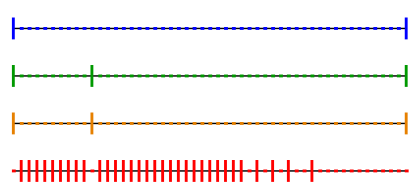

Figure 8. Performance of the three algorithms, and distribution of disk checkpoints, memory checkpoints and verifications (for the $\mathrm{A}_{D M V}$ algorithm) on platforms Hera and Coastal SSD with the HighLow pattern.

the large tasks at the beginning of the chain are more likely to fail, they will be checkpointed more often, as opposed to the small tasks at the end, which the algorithm does not even consider worth verifying.

HighLow pattern. Once again, we focus on platforms Hera and Coastal SSD. Similarly to Figure 7, Figure 8 assesses the impact of the HighLow pattern on the performance of the three algorithms as well as on the numbers and the positions of checkpoints and verifications. Recall that we set the first $10 \%$ of the tasks to contain $60 \%$ of the total computational weight, while the rest of the tasks contain the remaining $40 \%$. With 50 tasks and a total computational weight of $25000 \mathrm{~s}$, the first 5 tasks have a weight of $3000 \mathrm{~s}$ each, while the remaining tasks have a weight of around $222 s$ each. Under this configuration, an error occurring during the execution of a large task would $\operatorname{cost} T^{\text {lost }} \approx 1500 \mathrm{~s}$ time loss on average for fail-stop errors (see Equation (3)) and 3000s for silent errors, plus an additional 3000 s time loss for each preceding task that has not been checkpointed. With the MTBF on Hera, a large task will fail with probability $1.3 \%$, as opposed to the probability of $0.096 \%$ for small tasks. As a result, the disk checkpoint, which takes $300 \mathrm{~s}$, turns out to be still too expensive, but the memory checkpoint, which takes only $15.4 s$ on Hera, becomes mandatory: on average an error will occur way before the total accumulated cost of our preventive memory checkpoints even adds up to the cost of one task. On Coastal SSD, however, the memory checkpoint is still quite expensive, so that only one of the first 5 tasks is marked for verification and memory checkpointing. On both platforms, since the rest of the tasks are small, the solution is similar to the one we observed 
for the Uniform pattern, except that memory checkpoints and verifications are less frequent.

Summary of results. Overall, we observe that the combined use of disk checkpoints and memory checkpoints allows us to decrease the makespan, for the three task patterns and the four platforms. The use of partial verifications further decreases the makespan, especially on the Coastal SSD platform where the checkpointing costs are high. To give some numbers, our approach saves $2 \%$ of execution time on Hera and $5 \%$ on Atlas. These percentages may seem small, but they correspond to saving half an hour a day on Hera, and more than one hour a day on Atlas, with no further overhead.

\section{CONClusion AND Future Work}

In this paper, we proposed a two-level checkpointing scheme to cope with both fail-stop errors and silent data corruptions on large-scale platforms. Although numerous studies have dealt with either error source, few studies have dealt with both, while it is mandatory to address both sources simultaneously at scale. By combining standard disk checkpointing technique with in-memory checkpoints and verification mechanisms (partial or guaranteed), we have designed a multi-level dynamic programming algorithm that computes the optimal solution for a linear application workflow in polynomial time. Simulations based on realistic parameters on several platforms show consistent results, and confirm the benefit of the combined approach. While the most general algorithm has a high complexity of $O\left(n^{6}\right)$, where $n$ is the number of tasks, it executes within a few seconds for $n=50$ tasks, and therefore can be readily used for real-life linear workflows whose sizes rarely exceed tens of tasks.

One interesting future direction is to assess the usefulness of this approach on general application workflows. The problem gets much more challenging, even in the simplified scenario where each task requires the entire platform to execute. In fact, in this simplified scenario, it is already NP-hard to decide which task to checkpoint in a simple join graph $(n-1$ source tasks and a common sink task), with only fail-stop errors striking (hence a single level of checkpoint and no verification at all) [1]. Still, heuristics are urgently needed to address the same problem as in this paper, with two error sources, two checkpoint types and two verification mechanisms, if we are to deploy general HPC workflows efficiently at scale.

\section{ACKNOWLEDGMENTS}

This research was funded in part by the European project SCoRPiO, by the LABEX MILYON (ANR-10-LABX-0070) of Université de Lyon, within the program "Investissements d'Avenir" (ANR-11-IDEX-0007) operated by the French National Research Agency (ANR), and by the PIA ELCI project. Yves Robert is with Institut Universitaire de France. A short version of this paper was presented at PMBS'15 (without proceedings).

\section{REFERENCES}

[1] G. Aupy, A. Benoit, H. Casanova, and Y. Robert. Scheduling computational workflows on failure-prone platforms. In 17th Workshop on Advances in Parallel and Distributed Computational Models APDCM 2015, 2015.

[2] L. Bautista-Gomez, A. Benoit, A. Cavelan, S. K. Raina, Y. Robert, and H. Sun. Which verification for soft error detection? In Proc. HiPC, 2015. Full version available as INRIA RR-8741.

[3] L. Bautista Gomez and F. Cappello. Detecting silent data corruption through data dynamic monitoring for scientific applications. SIGPLAN Notices, 49(8):381-382, 2014.

[4] L. Bautista Gomez and F. Cappello. Detecting and correcting data corruption in stencil applications through multivariate interpolation. In Proc.1st Int. Workshop on Fault Tolerant Systems (FTS), 2015.

[5] L. Bautista-Gomez, S. Tsuboi, D. Komatitsch, F. Cappello, N. Maruyama, and S. Matsuoka. FTI: High performance fault tolerance interface for hybrid systems. In Proc. SC'11, 2011.

[6] A. Benoit, A. Cavelan, Y. Robert, and H. Sun. Assessing general-purpose algorithms to cope with fail-stop and silent errors. In Proc. PMBS'14, 2014. Full version available as INRIA-8599.

[7] A. Benoit, A. Cavelan, Y. Robert, and H. Sun. Optimal resilience patterns to cope with fail-stop and silent errors. In Proc. IPDPS'16, 2016. Full version available as INRIA RR-8786.

[8] A. Benoit, Y. Robert, and S. K. Raina. Efficient checkpoint/verification patterns. Int. J. High Performance Computing Applications, 2015.

[9] A. R. Benson, S. Schmit, and R. Schreiber. Silent error detection in numerical time-stepping schemes. Int. J. High Performance Computing Applications, 2014.

[10] E. Berrocal, L. Bautista-Gomez, S. Di, Z. Lan, and F. Cappello. Lightweight silent data corruption detection based on runtime data analysis for HPC applications. In Proc. HPDC, 2015.

[11] G. Bosilca, R. Delmas, J. Dongarra, and J. Langou. Algorithm-based fault tolerance applied to high performance computing. J. Parallel Distrib. Comput., 69(4):410-416, 2009.

[12] F. Cappello, A. Geist, B. Gropp, L. Kale, B. Kramer, and M. Snir. Toward Exascale Resilience. Int. Journal of High Performance Computing Applications, 23(4):374-388, 2009.

[13] F. Cappello, A. Geist, W. Gropp, S. Kale, B. Kramer, and M. Snir. Toward exascale resilience: 2014 update. Supercomputing frontiers and innovations, 1(1), 2014.

[14] A. Cavelan, S. K. Raina, Y. Robert, and H. Sun. Assessing the impact of partial verifications against silent data corruptions. In Proc. ICPP, 2015.

[15] K. M. Chandy and L. Lamport. Distributed snapshots: Determining global states of distributed systems. ACM Transactions on Computer Systems, 3(1):63-75, 1985.

[16] Z. Chen. Online-ABFT: An online algorithm based fault tolerance scheme for soft error detection in iterative methods. In Proc. PPoPP, pages 167-176, 2013.

[17] D. Fiala, F. Mueller, C. Engelmann, R. Riesen, K. Ferreira, and R. Brightwell. Detection and correction of silent data corruption for large-scale high-performance computing. In Proc. SC'12, page 78, 2012

[18] R. E. Lyons and W. Vanderkulk. The use of triple-modular redundancy to improve computer reliability. IBM J. Res. Dev., 6(2):200-209, 1962.

[19] A. Moody, G. Bronevetsky, K. Mohror, and B. R. d. Supinski. Design, Modeling, and Evaluation of a Scalable Multi-level Checkpointing System. In Proc. SC'10, 2010.

[20] T. O'Gorman. The effect of cosmic rays on the soft error rate of a DRAM at ground level. IEEE Trans. Electron Devices, 41(4):553-557, 1994.

[21] F. Quaglia. A cost model for selecting checkpoint positions in time warp parallel simulation. IEEE Trans. Parallel Dist. Syst., 12(4):346362, 2001

[22] P. Sao and R. Vuduc. Self-stabilizing iterative solvers. In Proc. ScalA '13, 2013

[23] S. Toueg and O. Babaoğlu. On the optimum checkpoint selection problem. SIAM J. Comput., 13(3), 1984.

[24] J. Ziegler, M. Nelson, J. Shell, R. Peterson, C. Gelderloos, H. Muhlfeld, and $\mathrm{C}$. Montrose. Cosmic ray soft error rates of 16-Mb DRAM memory chips. IEEE Journal of Solid-State Circuits, 33(2):246-252, 1998.

[25] J. F. Ziegler, H. W. Curtis, H. P. Muhlfeld, C. J. Montrose, and B. Chin. IBM experiments in soft fails in computer electronics. IBM J. Res. Dev., 40(1):3-18, 1996. 